\title{
Modeling Passenger-Flow in Real-Time Bus Tracking System
}

\author{
Bashir Shalaik, Ricky Jacob, and Adam C. Winstanley
}

\begin{abstract}
Transit networks in the real world are similar to data transfer across a computer network. In this paper, we present the similarity and differences between computer networks and transit networks. We have developed a passenger-flow simulation model and we tested the effects of transit services provided on passengers in term of delay and passenger quality of service. We present the passenger's behavior at bus stops, factors that affect passenger's interactions with buses, and the performance of buses. The objective of this research is to provide operators with improved metrics and better tools to manage the public transit network. In this paper, the overall transit network performance has been evaluated and summarized.
\end{abstract}

Index Terms-Bus tracking, passenger flow, quality of service, simulation.

\section{INTRODUCTION}

Computer networks and road networks have similarities. Most computer networks can be viewed as transportation infrastructures for the deployment of data. The aim of both networks is to move units/elements from an origin to destination as quickly as possible with no (or at least minimal) negative impact. In general, public transit buses work in a traffic environment especially in urban areas. The passenger flow in transit networks can be modeled and estimated using the service time, timetable $\&$ bus stop. For modeling the passenger flow, the bus stops and routes can be modeled as a network of nodes with passengers moving around the network. In packet switching computer networks, packets carry information related to their origin, destination and contents and keep moving between switches until they reach their final destination. The store-and forward mechanism is used for packet switching; when packets arrive at an input connection, switches store them into buffers before forwarding them to the next switch. If the buffers' capacity cannot keep up with the arrival rate of the packets, the switches become congested eyelids to packet ignorance.

The store-and forward concept [1] can be applied for long-journey passengers in public transit networks where passengers have to pass by different stops before reaching their final destinations. In this paper, we simulate the passenger flow through the transit network, examine the impact of services on passengers and test how passengers make use of transit network resources.

Manuscript received May 20, 2012; revised June 24, 2012

The authors are with the Department of Computer Science, National University of Ireland Maynooth, Ireland (e-mail: bsalaik@cs.nuim.ie; rjacob@cs.nuim.ie; adamw@cs.nuim.ie).

\section{COMPUTER NETWORKS}

Over the last twenty years, the field of computer networks has grown from simple to more advanced communication techniques [2]. The basic method used by computer networks is sending 'packets' of data through the connection media. This media consists of cables with hubs, switches and routers providing connection between the origins and destinations within the network. Most of the data networks operate on the concept of 'best effort delivery' where the network does not provide any guarantee that the data is delivered nor a user is given a guaranteed quality of service level or a guaranteed priority [3]. As in public transit networks, in a best effort network all users (passengers) obtain best effort services, they obtain unspecified variable bit rate and delivery time depending on the current load. In computer networks when a packet arrives at a router, route algorithms make on-the-fly decision and decide to which adjacent node the packet should be sent. These route decision-making protocols running in the routers use the information gathered about the network topology by the second group of protocols. The algorithms in route decision protocols become more and more complicated as the network grows. Computer networks provide congestion control using the Transmission Control Protocol (TCP). When the network is congested, the packets are lost or 'dropped'. Packet loss is normal in network operation and not be seen as a failure of network. Lost packet can be retransmitted by acknowledgment technique. When the network is congested, the sender reduces the rate at which data is sent as the packet loss was caused by the network congestion. Therefore, as in road networks, techniques and procedures are used to monitor and control the movement of packets through the network.

\section{PUBLIC TRANSIT NETWORKS}

Public transit networks have been defined as "networks of roads, streets, stops or any structure which permits either vehicular movement or flow of some commodity"[4]. Transportation networks can be classified based on this operational area such as urban or rural networks. Instead of packets, both vehicle and passengers can be considered as moving objects through the networks and the roads represent the main media on which vehicles are transported. People (Passengers) are transported on roads either in individual cars or automobiles or in mass transit/public transport by bus/coach (vehicle). In transportation networks, a road is defined as a one/return way loop that connects a sequence of geographical points called stops and terminals where passengers can board and alight. Many factors such as road 
length, number of stops and distance between stops can affect on transportation network structure [5]. During a bus journey, passengers go through different stages such as waiting, queuing and transferring from the origin point to the final destination. Services provided and the transportation network resources affect these different stages.

\section{COMPARISON BETWEEN PUBLIC TRANSIT NETWORKS AND COMPUTER NETWORKS}

There are some similarities and differences between transit networks and data networks in terms of moving objects, transfer mechanisms and monitoring procedures. The moving objects in each network can be seen as having remarkable similarity at different levels. In transportation networks, passengers are served individually as in data networks that divide data into small blocks (packet), which it sends individually. The store-and-forward process is used in networks so passengers and packets are waiting and queuing at bus stops/switches until they are transferred to the next point/node. Both passengers and packets are impacted by the network's performance such as delays and transmission rate. The movable units within a transport network are self-aware; that is they choose their route/destination, and control timing of the trip. On the other hand, data packets have no self-awareness and do not choose their origin/destination, nor do they choose their route. Propagation delay exists in both networks. In computer networks, delays caused by packets that pass through routers and switches due to software requirements need these devices to perform their function. In transportation networks, delay caused by increase of traffic demand that approaches the capacity of a road may cause traffic jam. The two networks handle congestion in different ways. While there are many instances where data packets are lost, this cannot be adopted for the traveling elements of transportation network. The best-effort delivery system cannot be adopted in transportation network so it is reasonable therefore to think that end-to-end route algorithms of computer network could be applied in transportation network [2]. For managing the traffic in transportation networks, the hold-and-express algorithm is used and on the other hand, in computer network store-and-forward algorithm is used to control data flow. The similarities of functionality of both networks in movable units from origin to destination points suggesting and encouraging adopting techniques and algorithms used in computer networks to model passengers flow through transportation networks.

\section{COMPUTER NETWORKS PERFORMANCE INDICATORS}

Most of a computer network's performance can be measured qualitatively and quantitatively. Several measures are used to evaluate a network's performance like- delay, throughput, delay-throughput product and jitters.

\section{A. Delay}

Delay refers to 'how long it takes for a bit of data to travel across the network from one computer to another' [1]; delay is measured in seconds or in fraction of seconds. Delays may differ slightly, depending on the location of the specific pair of computers that communicate. The data packets within computer networks are facing different type of delay which affects the overall performance. These type of delays are processing delay, that caused by node processing, queuing delay caused by the buffering pf packets at the station and transmission delay caused by media bandwidth.

\section{B. Throughput}

Throughput is the rate at which data can be sent through the network, and is usually specified in bits per seconds (bps). The throughput capability of the underlying hardware is called bandwidth.

\section{Delay-Throughput Product}

Delay-throughput product is the volume of data that can be present on the network. If we think of road analogy: when cars are entering a road at a fixed rate of $T$ cars per second and it take a car $D$ seconds to traverse road, the $T \times D$ additional cars will enter the road by the time the first car has made its complete trip.

\section{Jitter}

Jitter is the variance of the delay on the network.

\section{Quality OF SERVICES (QOS) IN TRANSIT NETWORKS}

Quality of services or QoS indicators is one of the common metrics that is used for public transit performance evaluation. QoS has been correlated with transit agencies profitability, productivity and passenger satisfaction [6]. Most of transit agencies measure their fleet services with respect to scheduled or planned headway, however many QoS indicators are being developed and used by transit services operators. The importance of reliable bus services to passengers is well known. Different metrics are used to measure network performance like- on-time performance, running time adherence, and headway adherence.

\section{A. On-Time Performance}

On-time performance refers to the level of success of the service (such as a bus or train) remaining on the published schedule. Many factors such as traffic, road accidents, and weather can have an impact on on-time performance.

\section{B. Running Time Adherence}

Running time refers to the time it takes vehicles to move from one location to another; running time adherence indicates how well vehicles move along a route.

\section{Headway Adherence}

Headway adherence measures the deviation in time spacing between vehicles, its computed as the actual headway minus scheduled headway. A metric such as Excess Waiting Time (EWT) is used to measure the additional wait experienced by passengers due to the irregular spacing of buses or those that failed to run.

\section{REAL-TIME BUS TRACKING SYSTEM FOR BLACKPOOL TRANSIT}

Bus tracking system (www.bustracking.co.uk) was a joint project between NUIM and Blackpool Transport. Various 
reliability measures were explored to visualize the behaviour of vehicles in ways to allow the operator to better assess and improve the quality of their public transit service [7]. The system uses off-the-shelf GPS/GPRS integrated units programmed to transmit location at regular intervals (45 seconds approximately) while the vehicle is in motion. The data is stored on a server and can be displayed through a standard web browser to show views representing current locations of vehicles in close-to-real-time. The system in Figure 1 displays real time locations of buses pictorially, textually and, using the facilities provided by the Microsoft Virtual Earth API, with 2D and 3D.

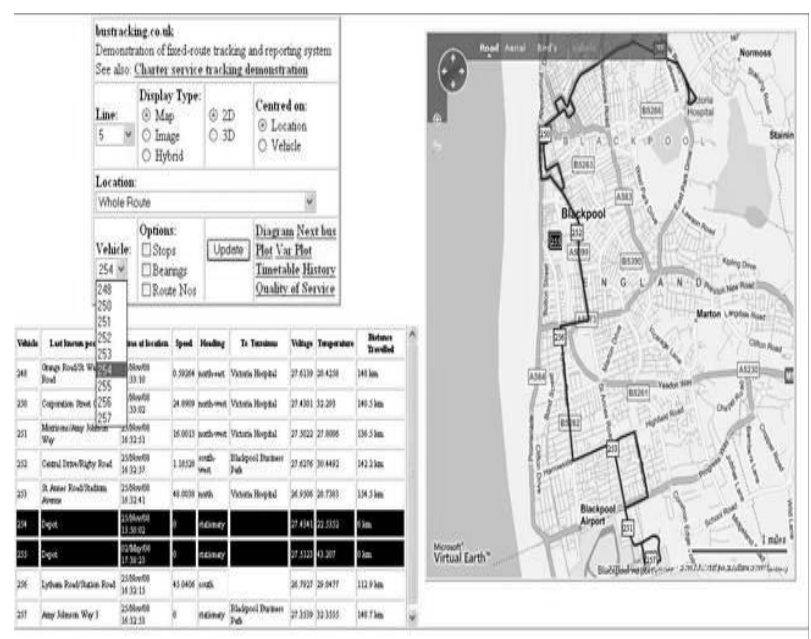

Fig. 1. The public interface of real-time bus-tracking system using Microsoft Virtual Earth.

Blackpool Transport Services operate the bus services in the city of Blackpool. For the purpose of modeling, analysis and evaluation of bus services in Blackpool, route 5, a high frequency route, was selected to be the test route. This bus route contains 73 bus stops in both directions, 14 of which are timing points (stops where a current time are specified in the timetable). Figure 2 shows the bus service on route 5 in Blackpool.

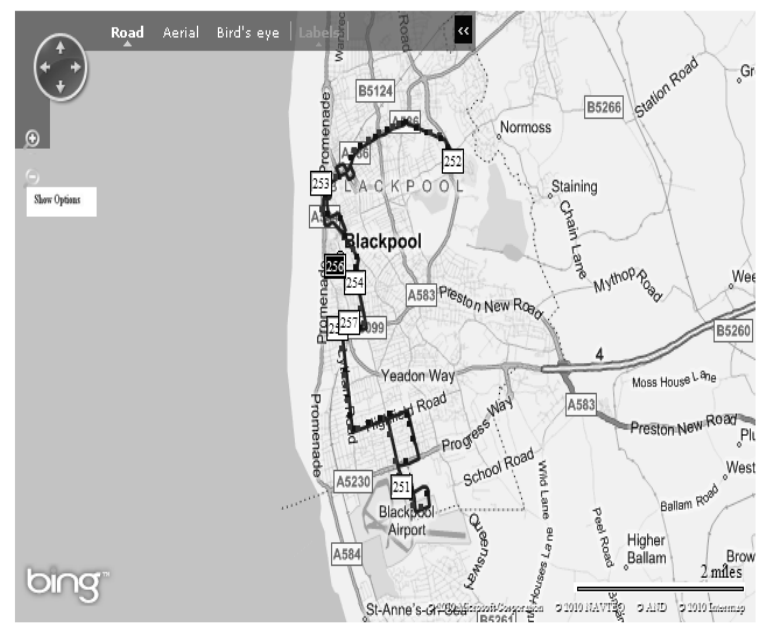

Fig. 2. Blackpool transport services bus route 5, blackpool city.

\section{A SiMUlATION MODEL AND EXPERIMENT}

The inadequate or infrequent collection of bus performance-indicator values brings forth the need for modeling and measuring these values. To identify the significant factors that have strong impacts on passenger trip time we have developed a simulation model. By modeling the passenger flow and measuring performance indicators, we can analysis the overall performance of the network and enable the operators to fix and maintain any problem that could occur. Passengers take a certain amount of time at bus stops waiting for the next bus, or queuing for boarding and alighting [8]. This time is called passenger service time and it is a function of number of passengers. A simulation model was developed to allow us to visualize what would happen at a transportation network under a variety of operating conditions. The object of this model is to analyse the overall results of passenger service time due to changes of some bus operational factors. The same set of variables and parameters were used for all the experiments. The initial conditions assumed the following:

1) Buses carry passengers up to their capacity,

2) Other passengers are waiting at bus stops, and

3) Buses are running according to their planned timetable and headways.

The proposed simulation can run different experiments with different scenarios that simulate passenger flow in a real-world environment with disruptions such as trip delays, miss-connection or cancellation of buses along a route.

There are three different levels or stages where passengers can be affected by transit services. These stages are: origin-point stage, boarding stage and arrival stage.

\section{A. Origin-Point Stage}

Origin point stage is where the passengers waits for the next bus with the possibility of deviation from advertised time table (delayed or ahead). At this stage passengers could have to wait longer than the expected time (Excess Waiting Time) due to irregular headway or bus that fails to run. When a bus arrives at a bus stop, there is a possibility that some or all passengers cannot board because of the bus's limited capacity and this will have a negative impact on their traveling time causing more delay and increasing waiting time at bus stops.

\section{B. Boarding Stage}

Boarding stage is the stage where the passenger services time (PST) is a function of passenger demand. Boarding passenger's wait-time must allow passengers in the bus to alight.

\section{Arrival Stage}

Arrival stage is the stage where passengers reach their final destination. Their arrival can be on time, ahead or delayed based on the deviation from timetable.

\section{RESUltS AND ANALYSIS}

In an ideal environment the passengers at a bus stop wait for their bus to arrive and the bus arrives on time and then letting some passengers (boarding passengers) on board to alight and passengers (demanding passengers) at the bus stop to board the bus respectively. This scenario is repeated at all bus stops until the bus reaches its final destination. 
Due to different factors such as traffic road, weather conditions or passengers demand, a bus can get delayed and deviates from planned the time-table and headway, and thus waiting or onboard passengers all along the bus route will be affected by this delay or deviation.

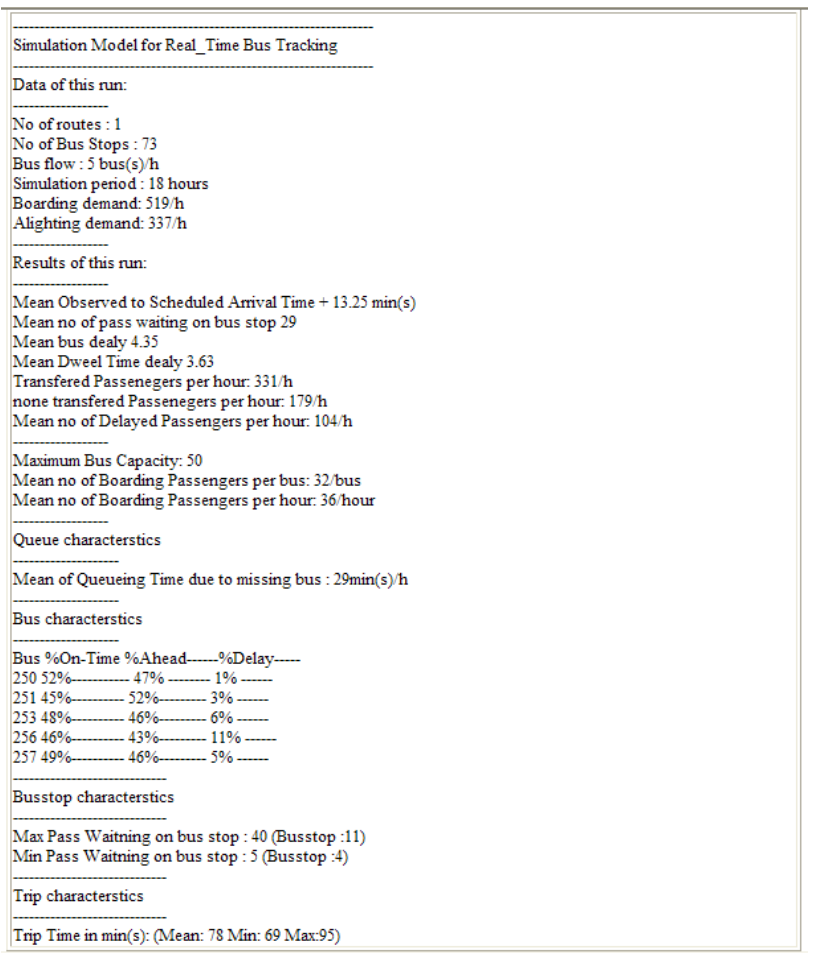

Fig. 3. Passenger-flow simulation output of the Real-time bus tracking system

From the simulations, the output transit network works with full capacity, and buses are following the timetable but there are some deviations as shown in Figure 3. Out of the overall demand, $65 \%$ of passenger transferred and $64 \%$ of bus capacity is used per hour. Buses seem to be running on their scheduled time that is 84 minutes. Mean running time is $78 \mathrm{~min}(\mathrm{~s})$. The factor of missed connections has a great impact on passengers travel time with average of $30 \mathrm{~min}(\mathrm{~s})$.

The significance of bus operation factors changes from case to case. For example, at the origin point stage, the bus departure has strong impact on passenger travel time as well as bus headway adherence.

\section{Conclusion}

The main goal of public transit networks is to provide timely, reliable and convenient transport services for passengers. Consequently, the most significant performance measure is the delay experienced by passengers. Passenger-based metrics together with bus-based metrics can give a more accurate and full description of bus transit-network performance. The modeled passenger flow simulation captures the performance of one-day of a public transit network and shows the significance of the factors such as bus delay or bus-misconnection can have on passenger travel-time.

\section{ACKNOWLEDGMENT}

Thanks are due to Blackpool Transport Ltd for facilitating this project and particularly to Oliver Howarth for his comments and feedback.

\section{REFERENCES}

[1] C. Douglas E. "Computer Networks and Internets with Internet Application," 4th Ed Prentice Hall, 2007, pp 83-98.

[2] S. Clement, N. Vogiatzis, M. Daniel, S. Wilson, and R. Clegg "Road Networks of the future: can Data Networks Help?" 28th Australasian Transport Research Forum.

[3] E. Tittel, “Computer Networking," Schaum's ouTlines 2002, pp. 122-125.

[4] Wikipedia, Definition of transport network. Available: http://en.wikipedia.org/wiki/Transport_network, March 2012.

[5] C. Yong-Zhou, L. Nan, and H. Da-Ren, "A study on some urban bus transport networks," Physica A: Statistical Mechanics and its Application, vol 376, Mar 2007, pp. 747-757

[6] J. Lin, P. Wang, and D. Barnum, "A Quality Control Framework for Bus Schedule Reliability", Transportation Research Part E-Transportation Review and Logistics, vol 44(6), 2007, pp 1086-1098

[7] A. Winstanley, B. Shalaik , J. Zheng, and R. Burke, "Visualizing Transport quality of Service," in Proc. GIS Research, UK (GISRUK 2009), pp 221-227.

[8] F. Rodrigo and T.Nick "Effect of passenger-Bus-Traffic Interaction on Bus Stop Operations," Transportation Planning and Technology, vol 28, Aug 2005, pp. 273-292

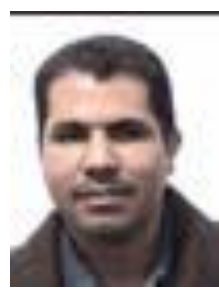

B. Shalaik is a Ph.D. student at National University of Ireland Maynooth, Bashir is supported by a PhD studentship from the Libyan Ministry of Education. His research interests are real-time public transport systems, transport data visualization, and advanced public transport systems Bashir received his BSc in computer science from Sebha University Libya, and his MSc in computer science from NUI Maynooth.

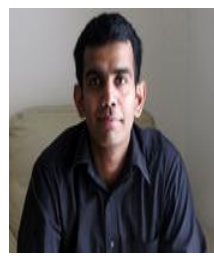

R. Jacob is a Ph.D. Student at the Department of Computer Science at the National University of Ireland Maynooth. He has a Masters degree in Spatial Information Technology from the University of Madras, Chennai, India. He has published his work in various conferences and journals related to Location Based Services and Geographical Information System. His current research interest lies in non-visual mobile spatial interaction by pedestrians to get spatial information using haptic feedback.

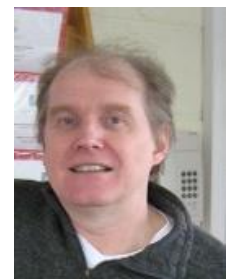

A. Winstanley is a Senior Lecturer and Head of the Department of Computer Science at Nationa University of Ireland Maynooth. He has a BA in archaeology from Cambridge University and an MSc and Ph.D. in Computer Science from Queen's University, Belfast. His research interests are in spatial databases, graphics recognition, navigation algorithms and transport information systems. 